\title{
Metacognitive self-reflectivity moderates the relationship between distress tolerance and empathy in schizophrenia
}

\author{
Kelsey A. Bonfilsa ${ }^{a}$, Kyle S. Minora ${ }^{a}$ Bethany L. Leonhardt ${ }^{b}$, and Paul H. Lysaker ${ }^{b, c,{ }^{*}}$ \\ aPsychology Department, Indiana University-Purdue University Indianapolis \\ bPsychiatry Department, Indiana University School of Medicine \\ cPsychiatry Department, Richard L. Roudebush VA Medical Center
}

\begin{abstract}
Deficits in empathy seen in schizophrenia are thought to play a major role in the social dysfunction seen in the disorder. However, little work has investigated potential determinants of empathic deficits. This study aimed to fill that gap by examining the effects of two variables on empathy - distress tolerance and metacognitive self-reflectivity. Fifty-four people with schizophrenia-spectrum disorders receiving services at an urban VA or community mental health center were assessed for empathy, metacognition, and distress tolerance. Bivariate correlations and moderation methods were used to ascertain associations amongst these variables and examine interactions. Results revealed that, against hypotheses, empathy was not related at the bivariate level to either distress tolerance or metacognitive self-reflectivity. However, consistent with hypotheses, moderation analyses revealed that participants with higher self-reflectivity showed no relationship between distress tolerance and empathy, while those with lower self-reflectivity showed a relationship such that reduced ability to tolerate distress predicted reduced empathy. Taken together, results of this study suggest that lack of distress tolerance can negatively affect empathy in people with schizophrenia with lesser capacity for metacognitive self-reflection; thus, fostering self-reflectivity may help overcome that negative impact. Future work is needed investigating the impact of metacognitively-tailored interventions on empathy in this population.
\end{abstract}

\section{Keywords}

schizophrenia-spectrum disorders; metacognition; social cognition; emotion regulation

\section{Introduction}

Empathy is a complex construct with a variety of physiological, cognitive, and affective processes. Empathy, a domain of social cognition, assists in forming a meaningful sense of others - their emotions, wishes and desires - and establishing intimate social connections (Davis, 1994). Empathic deficits have been broadly observed in schizophrenia (Bonfils et al.,

*Correspondence concerning this article should be addressed to Paul H. Lysaker, Roudebush VA Medical Center 1481 West 10th St., Indianapolis. IN 46202. plysaker@iupui.edu.

The authors declare no conflict of interest. 
2016b; Bonfils et al., 2017) and are thought to play a major role in the social dysfunction observed in this condition (Michaels et al., 2014; Shamay-Tsoory et al., 2007; Smith et al., 2014). Much research on empathy in schizophrenia investigates how reduced empathy might impact social outcomes, such as identifying facial emotional expressions (see meta-analysis by (Savla et al., 2013). Practically, if one cannot identify emotional expressions, the ability to empathize with the other is inherently hindered. However, problems identifying emotional expressions cannot fully explain deficits in empathy, which is a much more complex process (Decety and Jackson, 2004). Despite its importance, little work has investigated potential determinants of reduced empathy in schizophrenia. Thus, this study aimed to examine the impact of two constructs potentially related to empathy: distress tolerance and metacognitive self-reflectivity.

Distress tolerance refers to the ability to handle aversive emotional or physical experiential states (Simons and Gaher, 2005; Zvolensky et al., 2011) and is believed to be a key skill for human adaptation to stressful or emotional situations. Intuitively, people who are less able to tolerate aversive emotional experiences may also be less likely to empathically interact with someone in a situation that provokes difficult emotions. In these instances, the person may be hyper-focused on their own reaction and less able to provide an empathic response. A number of researchers have suggested that a failure to downregulate heightened aversive emotion could negatively impact empathy for people with schizophrenia (Bonfils et al., 2017; Cohen and Minor, 2010; Horan et al., 2006; Horan et al., 2015). Reduced distress tolerance has been broadly observed in mental disorders including borderline personality disorder (Gratz et al., 2006; Iverson et al., 2012; Linehan, 1993), eating disorders (Anestis et al., 2007; Hambrook et al., 2011), substance use disorders (Daughters et al., 2005; Dennhardt and Murphy, 2011), depression (Bernstein et al., 2011; Dennhardt and Murphy, 2011), and anxiety (Bernstein et al., 2011; Keough et al., 2010). More recently, decreased distress tolerance has also been found in schizophrenia-spectrum disorders (Chiappelli et al., 2014; Nugent et al., 2014). In schizophrenia, reduced distress tolerance has been correlated with increased psychotic symptoms, negative mood states (Stanage-Becker, 2009), reduced functioning, and increased cognitive deficits (Nugent et al., 2014).

To explore the effects of distress tolerance on empathy, this study examined the associations between these two variables in a sample of people with schizophrenia-spectrum disorders. We expected that reduced distress tolerance would negatively impact empathy; however, we also anticipated that this relationship may not exist for all people with schizophrenia. Indeed, recent literature has implicated metacognitive self-reflectivity as a potentially important construct for relationships relevant to social cognition. Metacognition was first seen as a term used in the education literature (Flavell, 1979), but has since been used variably in many literatures. In research on psychopathology, the term often implies a spectrum of psychological functions, referring to reflections about specific thoughts and feelings as well as more synthetic internal experiences, where thoughts, emotions, intentions, and how they interconnect are brought together to form integrated representations of self and others (Lysaker et al., 2013c; Semerari et al., 2003).

Metacognition as a construct seems to overlap with social cognition (of which empathy is one domain); indeed, the two constructs are related (Hasson-Ohayon et al., 2015). But, 
research shows that the two constructs are independent and differentially predict outcomes in schizophrenia (Hasson-Ohayon et al., 2015; Lysaker et al., 2013a). Thus, we may expect a bivariate correlation between metacognition and social cognitive constructs, such as empathy, but metacognition may also serve in a moderating role that impacts the relationship between variables such as distress tolerance and empathy. Indeed, metacognition has served in a similar role before - a recent study found that in the relationship between emotional awareness and self-esteem, metacognitive self-reflectivity moderated the relationship such that for those with low self-reflectivity, decreased awareness of emotions predicted reduced self-esteem, while at higher levels, the relationship was non-significant (Bonfils et al., 2016a). Metacognitive self-reflectivity may be of particular importance here, too, as opposed to other metacognitive components (awareness of the other, decentration, and mastery) because self-reflectivity, or the ability to understand and integrate one's own mental states (Lysaker et al., 2010), has potential to modify one's experience of internal distress, perhaps allowing understanding and adaptive reinterpretation of the experience of internal distress that accompanies reduced distress tolerance. This may be notable in the relationship between distress tolerance and empathy, as those with better ability to understand and make meaning from internal, self-oriented states (i.e., higher self-reflectivity) may be more able to be empathic than those with lesser capacity for self-reflectivity. Thus, we expected that those with increased metacognitive self-reflectivity would be able to overcome deficits in distress tolerance, causing the relationship between distress tolerance and empathy to become nonsignificant.

To examine these possibilities, we assessed empathy, distress tolerance, and metacognition and examined the following hypotheses: 1) Reduced empathy would be associated with lower distress tolerance and metacognitive self-reflectivity; and 2) Metacognitive selfreflectivity would moderate the relationship between distress tolerance and empathy such that the relationship would no longer be significant when relatively higher metacognitive self-reflectivity was present. Of note, to rule out the possibility that we were merely assessing emotion regulation, a construct sometimes linked with distress tolerance (Iverson et al., 2012; Simons and Gaher, 2005), we included a measure of emotion regulation as well and tested a similar moderation model.

\section{Method}

\subsection{Participants}

Participants included 56 people with schizophrenia-spectrum disorders confirmed by the Structured Clinical Interview for the Diagnostic Statistical Manual-IV (SCID-IV; (First et al., 2002). Two participants did not complete the entire interview, resulting in a sample of 54 participants for this study. Participants were recruited from an urban community mental health center or an outpatient psychiatry clinic of a of a Veterans' Affairs Medical Center. Clients were eligible to participate if they had not been hospitalized or experienced any medication changes in the prior month. Clients were not allowed to participate if chart review revealed a diagnosis of substance dependence, but current substance use or abuse did not preclude clients' participation in the study. 


\subsection{Measures}

2.2.1. Empathy.-Empathy was assessed using a single item from Heinrich's Quality of Life Scale (QOLS; Heinrichs et al., 1984). The QOLS is a 21-item measure completed by clinically trained research staff. Ratings are assigned following a semi-structured interview; higher scores suggest better functioning. The empathy item on the QOLS is a part of the Intrapsychic Foundations sub-score and assesses participants' capacity for empathic interaction. The QOLS is widely used, and the empathy item specifically has been used in prior studies (Barrio, 2001; Brekke and Barrio, 1997; Hooker et al., 2011; Lysaker et al., 2013b; Matsui et al., 2008). Studies have shown evidence of high inter-rater reliability of the QOLS (Lysaker et al., 2010) and validity (Cramer et al., 2000) in schizophrenia samples.

2.2.2. Distress tolerance.-The Distress Tolerance Scale (DTS; Simons and Gaher, 2005) contains 14 Likert-style items rated on a 5 -point scale from 1 , strongly agree, to 5 , strong disagree. The DTS can be averaged to create a total score; higher scores suggest increased ability to withstand emotional distress. An example item on the scale reads, "Feeling distressed or upset is unbearable to me." Research indicates the DTS has acceptable test-retest reliability, internal consistency, and construct validity (Simons and Gaher, 2005). In this sample, Cronbach's alpha suggested acceptable internal consistency for the DTS (0.82).

2.2.3. Metacognition.-The Metacognition Assessment Scale-Abbreviated (MAS-A; Lysaker et al., 2005) was used to assess metacognitive capacity. MAS-A scores were obtained from verbalizations produced as part of the Indiana Psychiatric Illness Interview (IPII; Lysaker et al., 2002), an interview asking for information about how individuals view their lives and mental illnesses in a semi-structured fashion. MAS-A ratings were assigned to transcribed IPII interviews. The MAS-A produces a total scores, as well as subscale scores in four domains, including self-reflectivity, awareness of the minds of others, decentration, and mastery (Lysaker et al., 2010). For this study, the focus was on the domain of self-reflectivity. The self-reflectivity subscale has scores ranging from 0-9. Higher scores reflect greater ability to think about and integrate one's own thoughts and emotions. Past work indicates the MAS-A has good validity (Lysaker et al., 2014) and internal consistency (Lysaker et al., 2007) in studies of those with schizophrenia.

2.2.4. Emotion regulation.-The Difficulties in Emotion Regulation Scale (DERS; Gratz and Roemer, 2004) contains 36 Likert-style items rated on a scale from 1, almost never, to 5, almost always. Items on the DERS can be summed to create a total emotion regulation score with higher scores reflecting poorer emotion regulation. An example reverse-scored item on the scale reads, "I pay attention to how I feel." Research has shown the DERS to have acceptable test-retest reliability, internal consistency, and construct validity in people with psychotic disorders (Owens et al., 2013). In this sample, Cronbach's alpha was acceptable for the total score (0.93).

2.2.5. Symptoms.-Symptoms were assessed using the Positive and Negative Syndrome Scale (PANSS; Kay et al., 1987). The PANSS has 30 items and is rated by clinically trained research staff based on a semi-structured interview. Two of five analytically derived factors

Psychiatry Res. Author manuscript; available in PMC 2019 July 01. 
were used in this study to control for symptoms typically seen in schizophrenia: Positive and Negative symptoms (Bell et al., 1994). The PANSS has been used widely in schizophrenia research and studies show evidence of validity and reliability for this measure (e.g., see Bell et al., 1994; Bell et al., 1992; Bryson et al., 1999).

\subsection{Procedures}

All procedures were approved by the relevant institutional review board. Participants in this study were part of a larger randomized controlled trial of Narrative Enhancement and Cognitive Therapy (NECT; Yanos et al., 2011). Participants' diagnoses were confirmed after their provision of informed consent. Master's-level research associates then completed baseline assessments (i.e., pre-intervention) with eligible participants. Only baseline data was used for this investigation. Raters for the MAS-A were blind to condition and other testing.

\subsection{Analyses}

Following descriptive statistics, bivariate correlations were conducted to address hypothesis one, that empathy would be positively associated with the ability to tolerate distress, emotion regulation, and self-reflectivity. To address hypothesis two, that self-reflectivity would moderate the relationship between distress tolerance and empathy, Hayes (2013) PROCESS macro conducted a series of ordinary least squares (OLS) regressions to assess moderation. Moderation was considered to be present if a model predicting empathy $(\mathrm{Y})$ with distress tolerance (X) and self-reflectivity (M) was improved by adding the interaction term (XM) to the model. Positive and negative symptoms were included as covariates to ensure a rigorous test when controlling for common symptoms of psychotic disorders. If moderation was found to be present, the interaction was examined in two ways: first, the data were graphed using the pick-a-point approach (Rogosa, 1980), enabling visualization of the relationship at different points of the moderator; second, the Johnson-Neyman technique (Bauer and Curran, 2005) identified the value of the moderator at which the relationship between distress tolerance and empathy changed significance. These procedures were repeated with emotion regulation in place of distress tolerance to assess if self-reflectivity moderates the relationship between emotion regulation and empathy.

\section{Results}

Participants were mostly male $(n=36,66.7 \%)$ and Black $(n=33,61.1 \%)$. The majority of participants had schizophrenia $(n=39,72.2 \%)$, and the remainder had schizoaffective disorder $(n=15,27.8 \%)$. Participants were middle-aged $(M=51.1)$, and had on average completed 12 years of school. The sample was non-acute, and symptoms reflected that, with evidence of moderate scores on the PANSS (Total $\mathrm{M}=70.2, \mathrm{SD}=13.2$; Positive $\mathrm{M}=15.2$, $\mathrm{SD}=4.2$; Negative $\mathrm{M}=16.1, \mathrm{SD}=5.8$ ).

Results of bivariate correlations can be seen in Table 1. Against expectations, empathy was not associated with either distress tolerance or emotion regulation. Self-reflectivity exhibited a positive, moderate correlation with empathy. Consistent with hypotheses, distress tolerance and emotion regulation were strongly correlated $(r=0.56)$, but not so strongly that we might

Psychiatry Res. Author manuscript; available in PMC 2019 July 01. 
suspect they were the same construct (i.e., $r<0.80$ ). Inability to tolerate distress also correlated with increased positive symptoms.

Results of moderation analyses can be seen in Table 2. For the model examining the relationship between distress tolerance and empathy, controlling for positive and negative symptoms, the interaction term was significant. The pick-a-point approach was used to graph this relationship (see Figure 1); results revealed that for those with lower levels of selfreflectivity, inability to tolerate distress negatively influenced empathy. At higher levels of self-reflectivity, distress tolerance had no effect on empathy, consistent with hypotheses. Specifically, the Johnson-Neyman value indicated participants at or below a value of 3.6 on the self-reflectivity scale ( $46 \%$ of the sample) had a significant relationship between distress tolerance and empathy, while those above 3.6 (54\%) reflected a non-significant relationship.

A similar moderation model was run to assess any moderating role of self-reflectivity in the relationship between emotion regulation and empathy. Though the overall model was significant, negative symptoms were the only significant predictor in the model (Table 2). The interaction term did not reach significance in this model, suggesting self-reflectivity does not moderate the relationship between emotion regulation and empathy in this data.

\section{Discussion}

Although much literature suggests that empathy, a construct key to our relationships and interpersonal connectedness, is impaired in schizophrenia, few studies have investigated factors that could contribute to that impairment. Accordingly, this study explored whether an interaction between relatively lower levels of distress tolerance and metacognitive selfreflectivity was linked to reduced empathy in schizophrenia. Counter to our hypotheses, no bivariate relationship was found between distress tolerance and empathy. However, in line with hypotheses, we found that this relationship becomes significant for participants with low metacognitive self-reflectivity - specifically, those who struggled to be able to name and reflect upon their own affective states. Ruling out the possibility that we would have detected a similar relationship with emotion regulation, we found that a similar moderating relationship did not exist for emotion regulation and empathy, supporting the independence of the constructs of distress tolerance and emotion regulation in schizophrenia.

Our result that there is no meaningful bivariate relationship between distress tolerance and empathy was surprising, and contrary to some other research suggesting a link between inability to tolerate distress and empathy or other social cognitive constructs (Bonfils et al., 2017; Cohen and Minor, 2010; Horan et al., 2006; Horan et al., 2015). However, in the literature, our result may align with some findings examining physiological evidence with empathy in schizophrenia. For example, one study found that skin conductance response in schizophrenia was associated with heightened personal distress, but not with self-reported empathy or other measures of social cognition (Ikezawa et al., 2012). Furthermore, attenuated event-related potential (ERP) response in people with schizophrenia during empathy-related paradigms has been found to be associated with increased feelings of personal distress, but not with self-reports of empathic tendencies (Corbera et al., 2014). 
Thus, our results may align well with this data and suggest these variables are not significantly related to one another.

Alternatively, in this sample, the lack of relationship could be explained by the interaction of self-reflectivity with distress tolerance; considering that only $46 \%$ of the sample had scores in the Johnson-Neyman region of significance, more than half evinced a non-significant relationship. Another possibility is that we see weaker correlations here due to disparities in measurement - distress tolerance was self-report while empathy was an observer rating. Past studies reflect that people with schizophrenia perceive that they are more empathic on an empathy questionnaire than do observers or informants (Bora et al., 2008; Lysaker et al., 2013b). In this study, participants may have reported their ability to tolerate distress in a similar fashion, reflecting greater abilities than may be observed by an external rater; such a pattern of reporting could have served to obfuscate findings. On the other hand, use of the empathy item from the HQOL scale here may assess levels of empathy expressed during interview, but could miss more nuanced aspects of empathy and how it may fluctuate when other internal experiences come into play - such as distress.

While bivariate relationships presented some surprises, moderation results aligned with expectations. Participants in this sample with higher self-reflectivity showed no relationship between distress tolerance and empathy, while those with lower self-reflectivity showed a relationship such that reduced ability to tolerate distress predicted reduced empathy. This relationship supports the theory put forth by several scholars suggesting that internal experiences of distress may interfere with social cognitive performance (Bonfils et al., 2017; Cohen and Minor, 2010; Horan et al., 2006; Horan et al., 2015); however, this only occurs when metacognitive self-reflectivity reflects a score of 3.6 or lower. On the MAS-A, this score suggests self-reflectivity is not adequately developed to the point where internal affect can be recognized or understood. This may suggest several things. First, it points to the possibility that the capacities to recognize and distinguish affective states from one another can be detangled from the ability to tolerate distress. Second, it may be that the ability to notice, distinguish and reflect upon affective states serves as a buffer for the effects of difficulties with distress tolerance. For example, it may be that among patients with lesser abilities to tolerate distress, the ability to name and understand experienced distress allows for empathy even when that distress is difficult to tolerate.

Of note, there are limitations. This was a cross-sectional study and causality cannot be inferred. In particular, alternative findings cannot be ruled out, including the possibility that empathy itself has effects on metacognitive capacity and ultimately through some interaction on distress tolerance. Our sample also was composed of mostly men in a later phase of illness who were enrolled in treatment. A different set of relationships might be observed among younger samples, women, or persons who refuse treatment. Longitudinal studies including more diverse participants are needed. We also included only single measures of our key constructs; future work is needed with multiple instruments assessing empathy, metacognition, and distress tolerance.

With replication, results from this study may have clinical implications. Interventions that enhance metacognition may help clients who have a low tolerance for distress to forge 
empathic relationships. One such intervention that has received recent attention is Metacognitive Reflection and Insight Therapy (MERIT; (Van Donkersgoed et al., 2014), which can be tailored to the metacognitive abilities of the client. In case work, this treatment has been linked to improvements in the abilities to understand the perspectives of others (Arnon-Ribenfield et al., In press; Buck and George, 2016; Hamm and Firmin, 2016). Results also suggest that distress tolerance and emotion regulation, though correlated, do not evince similar results for the moderation models tested here, suggesting the constructs may function independently in schizophrenia samples. Future work is needed examining these two constructs in a more nuanced fashion in this population.

\section{Acknowledgement}

Research reported in this publication was supported by the National Institute of Mental Health of the National Institutes of Health under award number 4R01MH094310-04. The content is solely the responsibility of the authors and does not necessarily represent the official views of the National Institutes of Health.

\section{References}

Anestis MD, Selby EA, Fink EL, Joiner TE, 2007 The multifaceted role of distress tolerance in dysregulated eating behaviors. Int. J. Eat. Disord 40 (8), 718-726. [PubMed: 17868125]

Arnon-Ribenfield N, Bloom R, Atzil-Sloman D, Peri T, de Jong S, Hasson-Ohayon I, In press Metacognitive Reflection and Insight Therapy (MERIT) among people with schizophrenia: Lessons from two case studies. Am. J. Psychother

Barrio C, 2001 Culture and schizophrenia: A cross-ethnic growth curve analysis. J. Nerv. Ment. Dis 189 (10), 676-684. [PubMed: 11708668]

Bauer DJ, Curran PJ, 2005 Probing interactions in fixed and multilevel regression: Inferential and graphical techniques. Multivariate Behav. Res 40 (3), 373-400. [PubMed: 26794689]

Bell MD, Lysaker PH, Beam-Goulet JL, Milstein RM, Lindenmayer JP, 1994 Five-component model of schizophrenia: assessing the factorial invariance of the Positive and Negative Syndrome Scale. Psychiatry Res 52 (3), 295-303. [PubMed: 7991723]

Bell MD, Milstein R, Beam-Goulet J, Lysaker P, Cicchetti D, 1992 The Positive and Negative Syndrome Scale and the Brief Psychiatric Rating Scale: reliability, comparability, and predictive validity. J. Nerv. Ment. Dis 180 (11), 723-728. [PubMed: 1431824]

Bernstein A, Marshall EC, Zvolensky MJ, 2011 Multi-method evaluation of distress tolerance measures and construct(s): Concurrent relations to mood and anxiety psychopathology and quality of life. J. Exp. Psychopathol 2 (3), 386-399.

Bonfils KA, Luther L, George S, Buck KD, Lysaker PH, 2016a The role of metacognitive selfreflectivity in emotional awareness and subjective indices of recovery in schizophrenia. J. Nerv. Ment. Dis 204 (12), 903-908. [PubMed: 27668353]

Bonfils KA, Lysaker PH, Minor KS, Salyers MP, 2016b Deficits in affective empathy in schizophrenia: A meta-analysis. Schizophr. Res 175, 109-117. [PubMed: 27094715]

Bonfils KA, Lysaker PH, Minor KS, Salyers MP, 2017 Empathy in schizophrenia: A meta-analysis of the Interpersonal Reactivity Index. Psychiatry Res 249, 293-303. [PubMed: 28142103]

Bora E, Gökçen S, Veznedaroglu B, 2008 Empathic abilities in people with schizophrenia. Psychiatry Res 160 (1), 23-29. [PubMed: 18514324]

Brekke JS, Barrio C, 1997 Cross-ethnic symptom differences in schizophrenia: The influence of culture and minority status. Schizophr. Bull 23 (2), 305-316. [PubMed: 9165639]

Bryson G, Bell M, Greig T, Kaplan E, 1999 Internal consistency, temporal stability and neuropsychological correlates of three cognitive components of the Positive and Negative Syndrome Scale (PANSS). Schizophr. Res 38 (1), 27-35. [PubMed: 10427608] 
Buck KD, George SE, 2016 Metacognitive Reflective and Insight Therapy for a person who gained maximal levels of metacognitive capacity and was able to terminate therapy. J. Contemp. Psychother 46 (4), 187-195.

Chiappelli J, Pocivavsek A, Nugent KL, Notarangelo FM, Kochunov P, Rowland LM, Schwarcz R, Hong LE, 2014 Stress-induced increase in kynurenic acid as a potential biomarker for patients with schizophrenia and distress intolerance. JAMA Psychiatry 71 (7), 761-768. [PubMed: 24806441]

Cohen AS, Minor KS, 2010 Emotional experience in patients with schizophrenia revisited: Metaanalysis of laboratory studies. Schizophr. Bull 36 (1), 143-150. [PubMed: 18562345]

Corbera S, Ikezawa S, Bell MD, Wexler BE, 2014 Physiological evidence of a deficit to enhance the empathic response in schizophrenia. Eur. Psychiatry 29 (8), 463-472. [PubMed: 24630376]

Cramer JA, Rosenheck R, Xu W, Thomas J, Henderson W, Charney DS, 2000 Quality of life in schizophrenia: A comparison of instruments. Schizophr. Bull 26 (3), 659-666. [PubMed: 10993404]

Daughters SB, Lejuez CW, Kahler CW, Strong DR, Brown RA, 2005 Psychological distress tolerance and duration of most recent abstinence attempt among residential treatment-seeking substance abusers. Psychol. Addict. Behav 19 (2), 208-211. [PubMed: 16011392]

Davis MH, 1994 Empathy: A social psychological approach Westview Press, Boulder, CO.

Decety J, Jackson PL, 2004 The functional architecture of human empathy. Behav. Cogn. Neurosci. Rev 3 (2), 71-100. [PubMed: 15537986]

Dennhardt AA, Murphy JG, 2011 Associations between depression, distress tolerance, delay discounting, and alcohol-related problems in European American and African American college students. Psychol. Addict. Behav 25 (4), 595-604. [PubMed: 21988480]

First MB, Spitzer RL, Gibbon M, Williams JBW, 2002 Structured Clinical Interview for DSM-IV-TR Axis I Disorders, Research Version, Patient Edition. (SCID-I/P). Biometrics Research, New York State Psychiatric Institute, New York, NY.

Flavell JH, 1979 Metacognition and cognitive monitoring: A new area of cognitive-developmental inquiry. Am. Psychol 34 (10), 906-911.

Gratz KL, Roemer L, 2004 Multidimensional assessment of emotion regulation and dysregulation: Development, factor structure, and initial validation of the Difficulties in Emotion Regulation Scale. J. Psychopathol. Behav. Assess 26 (1), 41-54.

Gratz KL, Rosenthal MZ, Tull MT, Lejuez CW, Gunderson JG, 2006 An experimental investigation of emotion dysregulation in borderline personality disorder. J. Abnorm. Psychol 115 (4), 850-855. [PubMed: 17100543]

Hambrook D, Oldershaw A, Rimes K, Schmidt U, Tchanturia K, Treasure J, Richards S, Chalder T, 2011 Emotional expression, self-silencing, and distress tolerance in anorexia nervosa and chronic fatigue syndrome. Br. J. Clin. Psychol 50 (3), 310-325. [PubMed: 21810109]

Hamm JA, Firmin RL, 2016 Disorganization and individual psychotherapy for schizophrenia: A case report of metacognitive reflection and insight therapy. J. Contemp. Psychother 46 (4), 227-234.

Hasson-Ohayon I, Avidan-Msika M, Mashiach-Eizenberg M, Kravetz S, Rozencwaig S, Shalev H, Lysaker PH, 2015 Metacognitive and social cognition approaches to understanding the impact of schizophrenia on social quality of life. Schizophr. Res 161 (2), 386-391. [PubMed: 25499045]

Hayes AF, 2013 Introduction to mediation, moderation, and conditional process analysis: A regressionbased approach Guilford Press, New York, NY.

Heinrichs DW, Hanlon TE, Carpenter WT, 1984 The Quality of Life Scale: An instrument for rating the schizophrenic deficit syndrome. Schizophr. Bull 10 (3), 388-398. [PubMed: 6474101]

Hooker CI, Bruce L, Lincoln SH, Fisher M, Vinogradov S, 2011 Theory of mind skills are related to gray matter volume in the ventromedial prefrontal cortex in schizophrenia. Biol. Psychiatry 70 (12), 1169-1178. [PubMed: 21917239]

Horan WP, Green MF, Kring AM, Nuechterlein KH, 2006 Does anhedonia in schizophrenia reflect faulty memory for subjectively experienced emotions? J. Abnorm. Psychol 115 (3), 496-508. [PubMed: 16866590]

Horan WP, Reise SP, Kern RS, Lee J, Penn DL, Green MF, 2015 Structure and correlates of selfreported empathy in schizophrenia. J. Psychiatr. Res 66-67, 60-66.

Psychiatry Res. Author manuscript; available in PMC 2019 July 01. 
Ikezawa S, Corbera S, Liu J, Wexler BE, 2012 Empathy in electrodermal responsive and nonresponsive patients with schizophrenia. Schizophr. Res 142 (1), 71-76. [PubMed: 23058162]

Iverson KM, Follette VM, Pistorello J, Fruzzetti AE, 2012 An investigation of experiential avoidance, emotion dysregulation, and distress tolerance in young adult outpatients with borderline personality disorder symptoms. Personal. Disord 3 (4), 415-422. [PubMed: 22452755]

Kay SR, Flszbein A, Opfer LA, 1987 The Positive and Negative Syndrome Scale (PANSS) for schizophrenia. Schizophr. Bull 13 (2), 261-276. [PubMed: 3616518]

Keough ME, Riccardi CJ, Timpano KR, Mitchell MA, Schmidt NB, 2010 Anxiety symptomatology: The association with distress tolerance and anxiety sensitivity. Behav. Ther 41 (4), 567-574. [PubMed: 21035619]

Linehan MM, 1993 Skills training manual for treating borderline personality disorder Guilford Press, New York, NY.

Lysaker PH, Carcione A, Dimaggio G, Johannesen J, Nicolò G, Procacci M, Semerari A, 2005 Metacognition amidst narratives of self and illness in schizophrenia: Associations with neurocognition, symptoms, insight and quality of life. Acta Psychiatr. Scand 112 (1), 64-71. [PubMed: 15952947]

Lysaker PH, Clements CA, Plascak-Hallberg CD, Knipscheer SJ, Wright DE, 2002 Insight and personal narratives of illness in schizophrenia. Psychiatry 65, 197-206. [PubMed: 12405078]

Lysaker PH, Dimaggio G, Buck KD, Carcione A, Nicolò G, 2007 Metacognition within narratives of schizophrenia: Associations with multiple domains of neurocognition. Schizophr. Res 93 (1), $278-$ 287. [PubMed: 17407806]

Lysaker PH, Dimaggio G, Daroyanni P, Buck KD, LaRocco VA, Carcione A, Nicolo G, 2010 Assessing metacognition in schizophrenia with the Metacognition Assessment Scale: Associations with the Social Cognition and Object Relations Scale. Psychol. Psychother 83 (3), 303-315. [PubMed: 20170602]

Lysaker PH, Gumley A, Luedtke B, Buck K, Ringer J, Olesek K, Kukla M, Leonhardt B, Popolo R, Dimaggio G, 2013a Social cognition and metacognition in schizophrenia: evidence of their independence and linkage with outcomes. Acta Psychiatr. Scand 127 (3), 239-247. [PubMed: 22967227]

Lysaker PH, Hasson-Ohayon I, Kravetz S, Kent JS, Roe D, 2013b Self perception of empathy in schizophrenia: Emotion recognition, insight, and symptoms predict degree of self and interviewer agreement. Psychiatry Res 206 (2-3), 146-150. [PubMed: 23246246]

Lysaker PH, Leonhardt BL, Pijnenborg M, van Donkersgoed R, de Jong S, Dimaggio G, 2014 Metacognition in schizophrenia spectrum disorders: Methods of assessment and associations with neurocognition, symptoms, cognitive style and function. Isr. J. Psychiatry Relat. Sci 51 (1), 54-62. [PubMed: 24858635]

Lysaker PH, Vohs JL, Ballard R, Fogley R, Salvatore G, Popolo R, Dimaggio G, 2013c Metacognition, self-reflection and recovery in schizophrenia. Future Neurol 8 (1), 103-115.

Matsui M, Sumiyoshi T, Arai H, Higuchi Y, Kurachi M, 2008 Cognitive functioning related to quality of life in schizophrenia. Prog. Neuropsychopharmacol. Biol. Psychiatry 32 (1), 280-287. [PubMed: 17884266]

Michaels TM, Horan WP, Ginger EJ, Martinovich Z, Pinkham AE, Smith MJ, 2014 Cognitive empathy contributes to poor social functioning in schizophrenia: Evidence from a new self-report measure of cognitive and affective empathy. Psychiatry Res 220 (3), 803-810. [PubMed: 25632418]

Nugent KL, Chiappelli J, Rowland LM, Daughters SB, Hong LE, 2014 Distress intolerance and clinical functioning in persons with schizophrenia. Psychiatry Res 220 (1), 31-36. [PubMed: 25107316]

Owens KA, Haddock G, Berry K, 2013 The role of the therapeutic alliance in the regulation of emotion in psychosis: An attachment perspective. Clin. Psychol. Psychother 20 (6), 523-530. [PubMed: 22570312]

Rogosa D, 1980 Comparing nonparallel regression lines. Psychol. Bull 88 (2), 307-321.

Savla GN, Vella L, Armstrong CC, Penn DL, Twamley EW, 2013 Deficits in domains of social cognition in schizophrenia: A meta-analysis of the empirical evidence. Schizophr. Bull 39 (5), 979-992. [PubMed: 22949733] 
Semerari A, Carcione A, Dimaggio G, Falcone M, Nicolo G, Procacci M, Alleva G, 2003 How to evaluate metacognitive functioning in psychotherapy? The Metacognition Assessment Scale and its applications. Clin. Psychol. Psychother 10 (4), 238-261.

Shamay-Tsoory SG, Shur S, Harari H, Levkovitz Y, 2007 Neurocognitive basis of impaired empathy in schizophrenia. Neuropsychology 21 (4), 431-438. [PubMed: 17605576]

Simons JS, Gaher RM, 2005 The Distress Tolerance Scale: Development and validation of a self-report measure. Motiv. Emotion 29 (2), 83-102.

Smith MJ, Horan WP, Cobia DJ, Karpouzian TM, Fox JM, Reilly JL, Breiter HC, 2014 Performancebased empathy mediates the influence of working memory on social competence in schizophrenia. Schizophr. Bull 40 (4), 824-834. [PubMed: 23770935]

Stanage-Becker EJ, 2009 Computerized experience sampling method in schizophrenia and schizoaffective disorder: Distress tolerance as a moderator of response to daily stressors, psychotic, and negative mood symptoms University of South Dakota.

Van Donkersgoed RJ, De Jong S, Van der Gaag M, Aleman A, Lysaker PH, Wunderink L, Pijnenborg G, 2014 A manual-based individual therapy to improve metacognition in schizophrenia: Protocol of a multi-center RCT. BMC Psychiatry 14 (1), 27-34. [PubMed: 24490942]

Yanos PT, Roe D, Lysaker PH, 2011 Narrative Enhancement and Cognitive Therapy: A new groupbased treatment for internalized stigma among persons with severe mental illness. Int. J. Group Psychother 61 (4), 576-595.

Zvolensky MJ, Leyro TM, Bernstein A, Vujanovic AA, 2011 Historical perspectives, theory, and measurement of distress tolerance, Distress tolerance: Theory, research, and clinical applications The Guilford Press, New York, NY, pp. 3-27.

Psychiatry Res. Author manuscript; available in PMC 2019 July 01. 


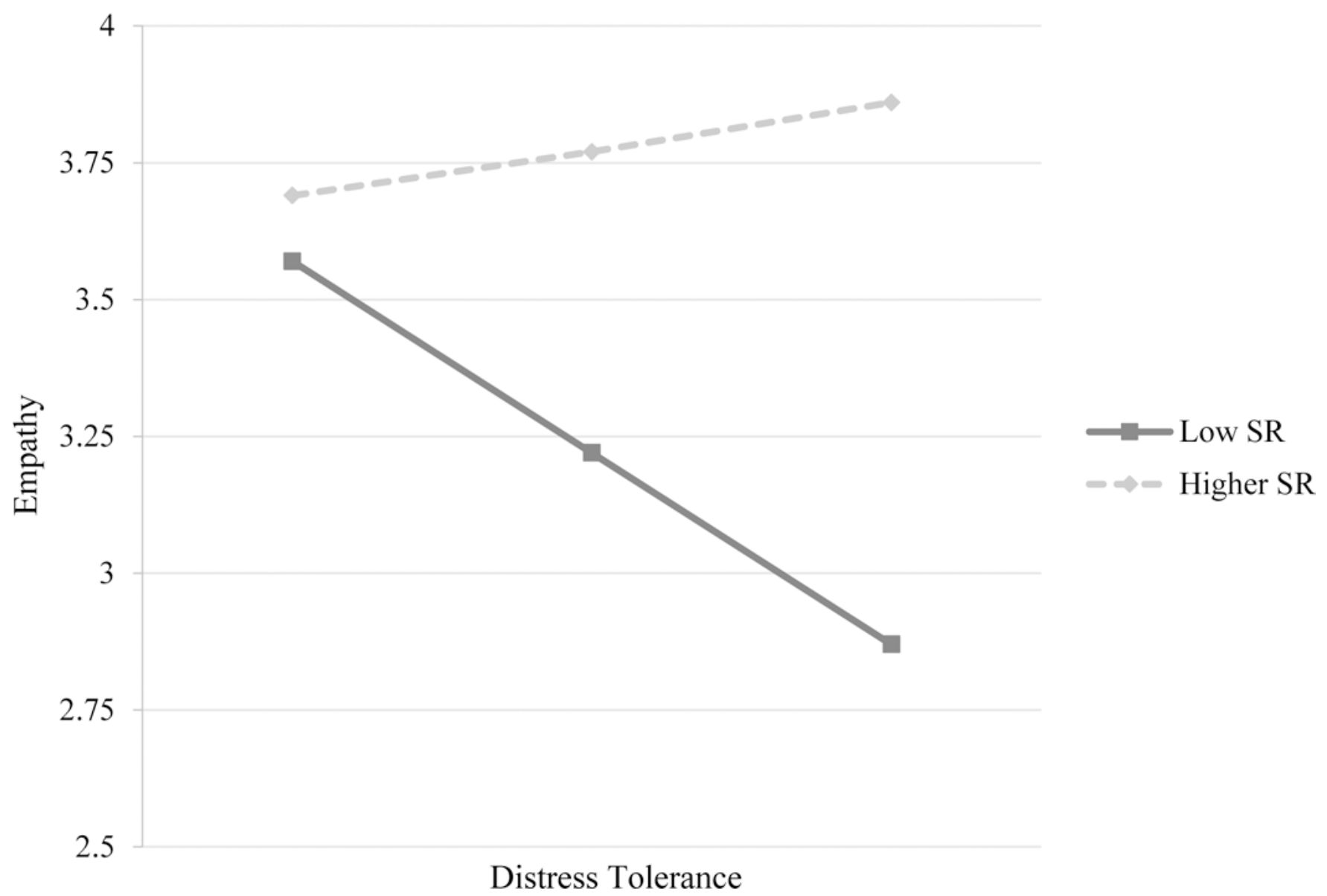

Figure 1.

Graph depicting the effect of distress tolerance on empathy at different levels of metacognitive self-reflectivity. SR = self-reflectivity. 
Table 1.

Correlations

\begin{tabular}{lllllll}
\hline & $\mathbf{1}$ & $\mathbf{2}$ & $\mathbf{3}$ & $\mathbf{4}$ & $\mathbf{5}$ & $\mathbf{6}$ \\
\hline 1. Empathy & 1 & & & & & \\
2. Distress Tolerance & -0.14 & 1 & & & & \\
3. Emotion Regulation & -0.09 & $0.56^{* *}$ & 1 & & & \\
4. Self-Reflectivity & $0.44^{* *}$ & 0.01 & 0.14 & 1 & & \\
5. PANSS positive & -0.15 & $0.32^{*}$ & 0.12 & -0.04 & 1 & \\
6. PANSS negative & $-0.48^{* *}$ & 0.02 & 0.08 & $-0.31^{*}$ & 0.25 & 1 \\
\hline
\end{tabular}

Note.

** Correlation is significant at the 0.01 level (2-tailed).

* Correlation is significant at the 0.05 level (2-tailed). 
Table 2.

Results of moderation analyses examining role of self-reflectivity.

\begin{tabular}{lrrrr}
\hline Variable & Coefficient & SE & $t$ & $p$ \\
\hline Model 1 (Distress Tolerance): & $R^{2}=0.39, F=6.26, p<0.01$ \\
Intercept & 6.56 & 1.36 & 4.83 & $<0.001$ \\
Self-Reflectivity & -0.39 & 0.28 & -1.36 & 0.181 \\
Distress Tolerance & -0.06 & 0.03 & -2.30 & 0.026 \\
Interaction Term & 0.01 & 0.01 & 2.04 & 0.047 \\
PANSS Positive & 0.01 & 0.03 & 0.35 & 0.726 \\
PANSS Negative & -0.06 & 0.02 & -3.17 & 0.003 \\
Model 2 (Emotion Regulation): $R^{2}=0.26, F=3.50, p=0.009$ \\
Intercept & 4.83 & 1.60 & 3.02 & 0.004 \\
Self-Reflectivity & -0.08 & 0.39 & -0.21 & 0.836 \\
Emotion Regulation & -0.01 & 0.02 & -0.79 & 0.435 \\
Interaction Term & 0.00 & 0.00 & 0.70 & 0.490 \\
PANSS Positive & -0.01 & 0.03 & -0.24 & 0.810 \\
PANSS Negative & -0.05 & 0.02 & -2.21 & 0.032 \\
\hline
\end{tabular}

Psychiatry Res. Author manuscript; available in PMC 2019 July 01. 